\title{
EFFECT OF INITIAL STRESS ON THE DYNAMIC RESPONSE OF A MULTI-LAYERED PLATE-STRIP SUBJECTED TO AN ARBITRARY INCLINED TIME-HARMONIC FORCE
}

\author{
A. DASSDEMIR \\ Department of Mathematics, Faculty of Arts and Sciences \\ Kastamonu University \\ Kastamonu, TURKEY \\ E-mail: ahmetdasdemir37@gmail.com
}

\begin{abstract}
The forced vibration of a multi-layered plate-strip with initial stress under the action of an arbitrary inclined time-harmonic force resting on a rigid foundation is considered. Within the framework of the piecewise homogeneous body model with the use of the three-dimensional linearized theory of elastic waves in initially stressed bodies (TLTEWISB), a mathematical modelling is presented in plane strain state. It is assumed that there exists the complete contact interaction at the interface between the layers and the materials of the layer are linearly elastic, homogeneous and isotropic. The governing system of the partial differential equations of motion for the considered problem is solved approximately by employing the Finite Element Method (FEM). Further, the influence of the initial stress parameter on the dynamic response of the plate-strip is presented.
\end{abstract}

Key words: multi-layered plate-strip, finite element method, initial stress, time-harmonic force.

2010 Mathematics Subject Classification: 74H15; 74S05.

\section{Introduction}

Due to the application of an external force such as mechanical or thermal loads, elastic solid materials are deformed, and they go back to their original shape after the force has been applied. Deformations in bodies are investigated in terms of the stresses and displacements components. Corresponding problems are encountered in almost all areas of applied sciences and engineering such as wave propagations, finite deformation theories or the diffraction theory. Hence, the subject "deformation of solids" has been under intense interest by many researchers.

Problems regarding non-linear effects in the dynamics of the layered elastic systems depend significantly on many factors. Two of the mentioned factors are the following ones: (a) the frequency response of the body under consideration, (b) the static initial stresses in each layer in which they exist before the application of the external dynamical force. The factor (a) is one of the most decisive features of the dynamic behavior of the system. The initial stresses in the layers of the body mentioned in the factor (b) may arise due to technological requirements or the environmental temperature. Note that the influence of the initial stresses on the dynamical behavior of the multi-layered body cannot be investigated within the framework of the classical linear theory of elastodynamics due to the fact that the initial stress displays nonlinear effects. However, the corresponding investigations can be made within the scope of the threedimensional linearized theory of elastic waves in initially stressed bodies (TLTEWISB). Note that these are based on the two fundamental assumptions such as (i) the pre-stressed state (or initial stress-state) is exactly homogeneous and static; and (ii) the additional dynamic loading subjected to the pre-stressed body is significantly smaller than the magnitude of the initial loading. The monographs $[1,2]$ give detailed information on the subject. Some examples of studies made on the influence of initial stresses on the multilayered system are given in [3-10]. 
In this paper, a mathematical modelling is presented to investigate the influence of the initial stress on the frequency response of a multi-layered plate-strip under the action of an arbitrary inclined timeharmonic external force resting on a rigid foundation. Note that the investigation is carried out within the framework of the piecewise homogeneous body model by the use of the TLTEWISB. Furthermore, the problem under consideration is approximately solved by employing the FEM, and certain numerical results are given. In particular, the influence of the initial stress on the dynamic response of the plate-strip is investigated.

\section{Problem formulation}

Consider a multi-layered pre-stressed plate-strip with the length $2 a$ and the height $h$ resting on a rigid foundation. The body has linear elastic, homogenous and isotropic layers. The height of each layer is denoted by $h^{(r)}$, where $r=1,2, \ldots, m$. As can be seen from Fig.1, an arbitrary inclined time-harmonic linear load is applied to the midpoint of the free surface of the plate-strip. It is assumed that the Cartesian coordinates denoted by $x_{i}$ in the natural state coincide with the Lagrange coordinates. It should be noted that before compounding each layer with one another and with the rigid foundation, each layer is separately subjected to a uniaxial uniformly distributed normal external force. This may be a stretching force or a compressing force. Consequently, an initial stress arises in each layer. These initial stresses are determined by utilizing the linear theory of elasticity as follows

$$
\sigma_{11}^{(r), 0} \geq 0 \quad \text { and } \quad \sigma_{i j}^{(r), 0}=0 \quad \text { for } \quad i j \neq 11
$$

where $i ; j=1,2$ and $\sigma_{11}^{(r), 0}$ denotes the unique non-zero initial stress of the $r$ - th layer. The values relating to the corresponding layer of the plate-strip are denoted by the superscript " $(r)$ ". Furthermore, the values relating to the initial state are denoted by the additional upper index " 0 ". According to Fig.1, the considered plate-strip includes the domain $D=\bigcup_{r=1}^{m} D^{(r)}$, where

$$
D^{(r)}=\left\{\left(x_{1}, x_{2}\right):-a \leq x_{1} \leq a, h^{\overline{(r)}} \leq x_{2} \leq h^{\overline{(r-1)}}\right\} .
$$

In Eq.(2.2), the notation $h^{\overline{(r)}}=-\sum_{l=1}^{r} h^{(l)}$ is used.

According to Guz [1-2], the equations of motion of the TLTEWISB are

$$
\sigma_{i j, j}^{(r)}+\sigma_{11}^{(r), 0} u_{i, 11}^{(r)}=\rho^{(r)} \ddot{u}_{i}^{(r)}, \quad i ; j=1,2 .
$$

In Eq.(2.3), $\rho^{(r)}$ is the mass density of the $r$-th material in the natural state, $u_{i}^{(r)}$ are the components of the displacements tensor, and $\sigma_{i j}^{(r)}$ are the components of the stress tensor. The dot over the quantities is time differentiation and the subscripts followed by a comma indicate the space-coordinate differentiation. Here and below, the repeated index in the subscript is summed with respect to that index.

For an isotropic compressible material, the mechanical and geometrical relations under consideration can be given as 


$$
\sigma_{i j}^{(r)}=\lambda^{(r)} \varepsilon_{\ell \ell}^{(r)} \delta_{i j}+2 \mu^{(r)} \varepsilon_{i j}^{(r)}, \quad \varepsilon_{i j}^{(r)}=\left(u_{i, j}^{(r)}+u_{j, i}^{(r)}\right) / 2
$$

where $\lambda^{(r)}$ and $\mu^{(r)}$ are the Lamé constants, $\delta_{i j}$ is the Kronecker delta, and $\varepsilon_{i j}^{(r)}$ are the components of the strain tensor.

According to the foregoing assumptions, there exist the following boundary-contact conditions

$$
\begin{array}{ll}
\left.\sigma_{21}^{(l)}\right|_{x_{2}=0}=-p_{0} \delta\left(x_{1}\right) \mathrm{e}^{\mathrm{i} \omega t} \cos \alpha, & \left.\sigma_{22}^{(l)}\right|_{x_{2}=0}=-p_{0} \delta\left(x_{1}\right) \mathrm{e}^{\mathrm{i} \omega t} \sin \alpha, \\
\left.\sigma_{i 2}^{(r+1)}\right|_{x_{2}=h} \overline{(r)}=\left.\sigma_{i 2}^{(r)}\right|_{x_{2}=h} \overline{(r)}, & \left.u_{i}^{(r+1)}\right|_{x_{2}=h} \overline{(r)}=\left.u_{i}^{(r)}\right|_{x_{2}=h} \overline{(r)}, \\
\left.\left(\sigma_{11}^{(r), 0} u_{j, 1}^{(r)}+\sigma_{1 j}^{(r)}\right)\right|_{x_{1}= \pm a}=0, & \left.u_{j}^{(m)}\right|_{x_{2}=-h}=0
\end{array}
$$

where $\delta(\cdot)$ is the Dirac delta function.

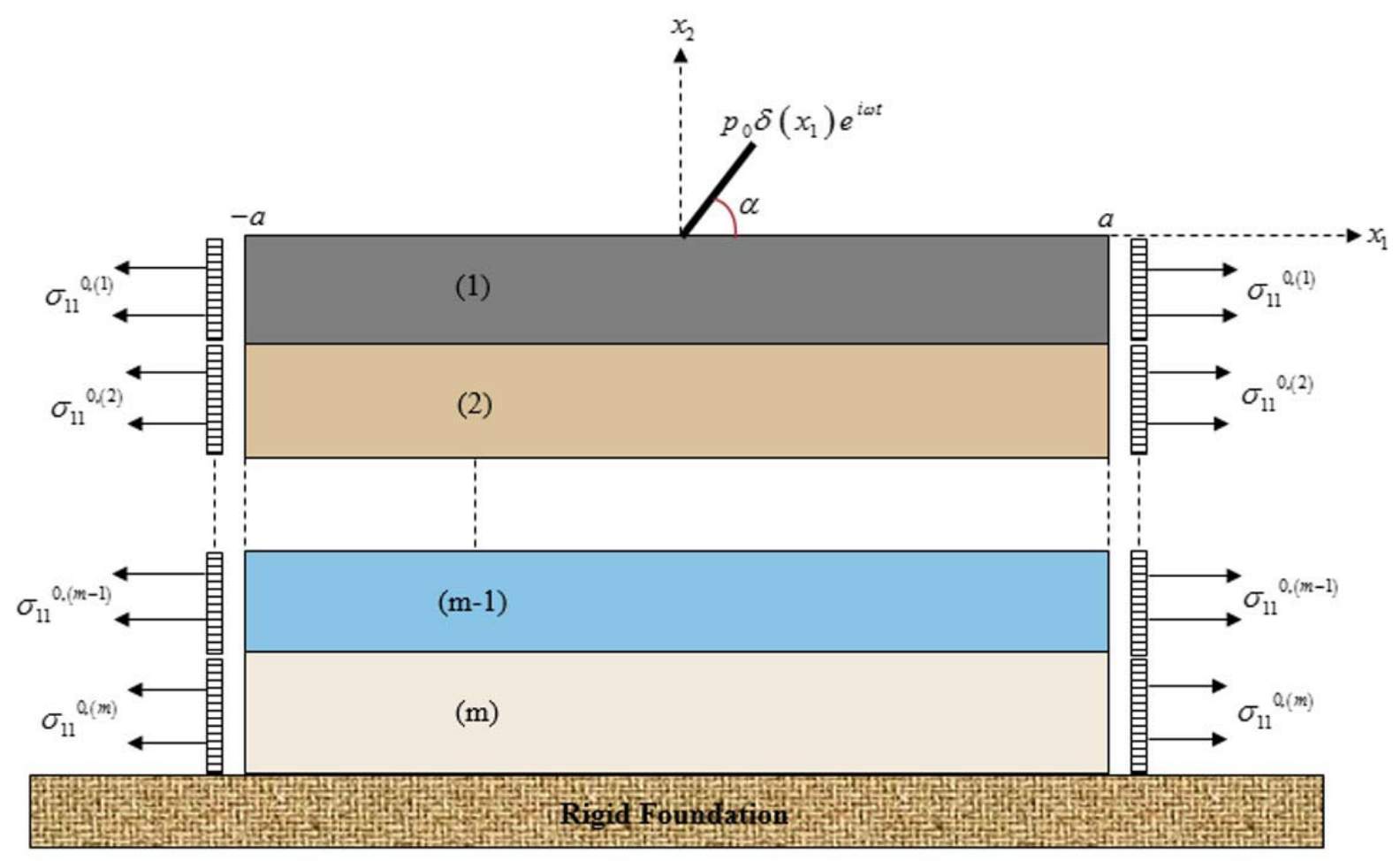

Fig.1a. Elastic multi-layered structure. 


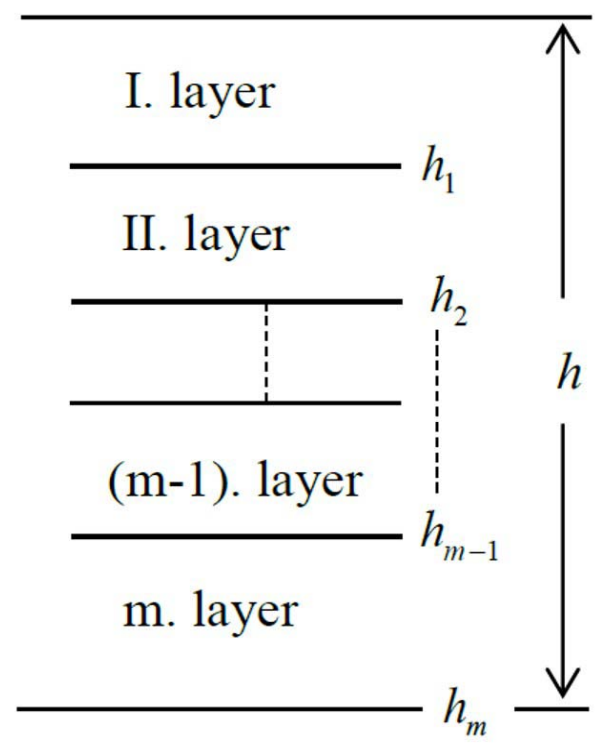

Fig.1b. Scheme of heights of layers.

\section{Solution procedure}

Since the external linear load is time-harmonic, with frequency $\omega$, as $p_{0} \delta\left(x_{1}\right) e^{i \omega t}$, all the dependent variables of the problem can be written in the form

$$
\left\{u_{i}^{(r)}, \sigma_{i j}^{(r)}, \varepsilon_{i j}^{(r)}\right\}\left(x_{1}, x_{2}, t\right)=\left\{\tilde{u}_{i}^{(r)}, \tilde{\sigma}_{i j}^{(r)}, \tilde{\varepsilon}_{i j}^{(r)}\right\}\left(x_{1}, x_{2}\right) \mathrm{e}^{\mathrm{i} \omega t} .
$$

In addition, introduce the dimensionless coordinate system

$$
\hat{x}_{1}=\frac{x_{1}}{h} \quad \text { and } \quad \hat{x}_{2}=\frac{x_{2}}{h} .
$$

Hence, applying the expression in Eq.(3.1) after the coordinate transformation in Eq.(3.2) into the foregoing equations and boundary-contact conditions, the same equations are obtained directly for the amplitude of the sought values by replacing the terms $\partial^{2} u_{j}^{(r)} / \partial t^{2}$ and $p_{0} \delta\left(x_{l}\right) e^{i \omega t}$ with $-\omega^{2} u_{j}^{(r)}$ and $p_{0} \delta\left(x_{1}\right)$, respectively. Clearly, they are as follows

$$
\begin{aligned}
& \hat{D}^{(r)}=\left\{\left(\hat{x}_{1}, \hat{x}_{2}\right):-a / h \leq \hat{x}_{1} \leq a / h, \quad h^{\overline{(r)}} / h \leq \hat{x}_{2} \leq h^{\overline{(r-l)}} / h\right\}, \\
& \tilde{\sigma}_{i j, j}^{(r)}+\sigma_{0}^{(r), 0} \tilde{u}_{i, l 1}^{(r)}+\rho^{(r)} \omega^{2} h^{2} \tilde{u}_{i}^{(r)}=0, \\
& \left.\tilde{\sigma}_{21}^{(l)}\right|_{\hat{x}_{2}=0}=-p_{o} \delta\left(h \hat{x}_{1}\right) \cos \alpha,\left.\quad \tilde{\sigma}_{22}^{(l)}\right|_{\hat{x}_{2}=0}=-p_{o} \delta\left(h \hat{x}_{1}\right) \sin \alpha, \\
& \left.\tilde{\sigma}_{i 2}^{(r+l)}\right|_{\hat{x}_{2}=h^{(r)} / h}=\left.\tilde{\sigma}_{i 2}^{(r)}\right|_{\hat{x}_{2}=h^{(r)} / h},\left.\quad \tilde{u}_{i}^{(r+1)}\right|_{\hat{x}_{2}=h^{(r)} / h}=\left.\tilde{u}_{i}^{(r)}\right|_{\hat{x}_{2}=h^{(r)} / h},
\end{aligned}
$$




$$
\left.\left(\sigma_{11}^{(r), 0} \tilde{u}_{j, l}^{(m)}+\tilde{\sigma}_{1 j}^{(r)}\right)\right|_{\hat{x}_{1}= \pm a / h}=0,\left.\quad \tilde{u}_{j}^{(2)}\right|_{\hat{x}_{2}=-1}=0
$$

For simplicity, all the superimposed notations will be omitted after this unless specified otherwise.

Since the geometry of the problem is a quite complex, an analytical solution to the problem cannot be obtained. Hence, the problem is approximately solved by employing the FEM. According to the wellknown procedure, multiplying equations of motion in (3.4) with by - multiply by the test functions $v_{1}=v_{1}\left(x_{1}, x_{2}\right)$ and $v_{2}=v_{2}\left(x_{1}, x_{2}\right)$, summing them side-by-side, integrating the last equation over the domain $D$ and after certain mathematical manipulations,

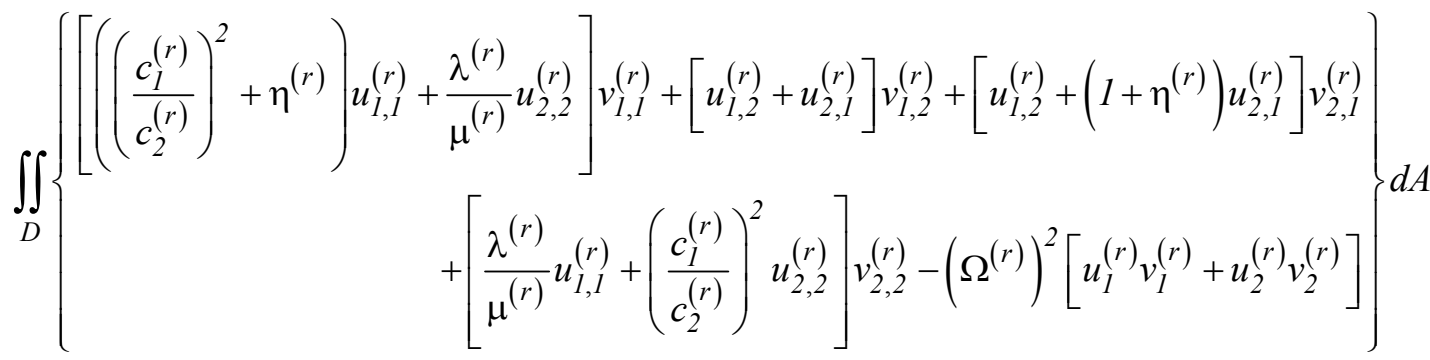

$$
\begin{aligned}
& =\int_{-a}^{a}-\left.p_{0} \frac{\delta\left(x_{1}\right)}{\mu^{(I)}}\left(v_{I}^{(I)} \cos \alpha+v_{2}^{(I)} \sin \alpha\right)\right|_{x_{2}=0} d x_{I}
\end{aligned}
$$

is obtained. In Eq.(3.8), the following notations are used

$$
c_{1}^{(r)}=\sqrt{\lambda^{(r)}+2 \mu^{(r)} / \rho^{(r)}}, \quad c_{2}^{(r)}=\sqrt{\mu^{(r)} / \rho^{(r)}}, \quad \Omega^{(r)}=\frac{\omega h}{c_{2}^{(r)}} \quad \text { and } \quad \eta^{(r)}=\sigma_{11}^{(r), 0} / \mu^{(r)}
$$

where $c_{1}^{(r)}$ is the speed of dilatation waves, $c_{2}^{(r)}$ is the speed of distortion wave, $\Omega^{(r)}$ is the dimensionless frequency, and $\eta^{(r)}$ is the initial stress parameter, respectively.

Denoting the terms in the left and right side of Eq.(3.8), respectively, by $B\left(\boldsymbol{u}^{(r)}, v^{(r)}\right)$ and $l\left(v^{(r)}\right)$, the total energy functional $J\left(\boldsymbol{u}^{(r)}\right)=B\left(\boldsymbol{u}^{(r)}, \boldsymbol{u}^{(r)}\right) / 2-l\left(\boldsymbol{u}^{(r)}\right)$ is obtained as follows

$$
\begin{aligned}
& J\left(\boldsymbol{u}^{(r)}\right)=\frac{1}{2} \iint_{D}\left\{\begin{array}{l}
\left(\frac{c_{1}^{(r)}}{c_{2}^{(r)}}\right)^{2}\left[\left(\frac{\partial u_{1}^{(r)}}{\partial x_{1}}\right)^{2}+\left(\frac{\partial u_{2}^{(r)}}{\partial x_{2}}\right)^{2}\right]+2 \frac{\lambda^{(r)}}{\mu^{(r)}} \frac{\partial u_{1}^{(r)}}{\partial x_{1}} \frac{\partial u_{2}^{(r)}}{\partial x_{2}}+\left[\frac{\partial u_{1}^{(r)}}{\partial x_{2}}+\frac{\partial u_{2}^{(r)}}{\partial x_{1}}\right]^{2}+ \\
+\eta^{(r)}\left[\left(\frac{\partial u_{1}^{(r)}}{\partial x_{1}}\right)^{2}+\left(\frac{\partial u_{2}^{(r)}}{\partial x_{1}}\right)^{2}\right]-\left(\Omega^{(r)}\right)^{2}\left[\left(u_{1}^{(r)}\right)^{2}+\left(u_{1}^{(r)}\right)^{2}\right]
\end{array}\right\} d A+ \\
& +\left.\int_{-a}^{a} p_{0} \frac{\delta\left(x_{1}\right)}{\mu^{(l)}}\left(u_{1}^{(l)} \cos \alpha+u_{2}^{(l)} \sin \alpha\right)\right|_{x_{2}=0} d x_{1} .
\end{aligned}
$$


As known from the principle of calculus of variation, equating the first variational of the total energy functional $J\left(\boldsymbol{u}^{(\boldsymbol{r})}\right)$ given in Eq.(3.10) to zero, the equations of motion and the corresponding boundarycontact conditions under consideration are obtained. In this way, the validity of the functional (3.10) is proven.

According to the virtual work principle and the standard Rayleigh-Ritz method, the domain $D$ is divided into a number of sub-domains. It should be noted that the number of these sub-domains is determined following the requirements such that the boundary conditions must be satisfied with very high accuracy and numerical results obtained must converge sufficiently well. Now the displacement components are represented at the nodes as

$$
u_{1}^{(k)}=\sum_{i=1}^{M} a_{i}^{(k)} N_{i}(t, s) \quad \text { and } \quad u_{2}^{(k)}=\sum_{i=1}^{M} b_{i}^{(k)} N_{i}(t, s)
$$

where $M$ is the number of the nodes over the $k$-th element, $a_{i}$ and $b_{i}$ are the unknown coefficients that need to be determined, $N_{j}(t, s)$ represents the shape functions over the $k$ - th element, and $t$ and $s$ are its local normalized coordinate components in the local coordinate system associated with the corresponding element. It should be pointed out that the shape functions $N_{j}(t, s) \in L_{2}^{l}$, where $L_{2}^{l}$ represents a set of the functions such as the squares of them and their first order partial differentials are integrable in the sense of Lebesque. Each of the shape functions $N_{j}(t, s)$, on the other hand, is defined over the domain $[-1,1] \times[-1,1]$, and the list of them can be found in [11].

Substituting the approximate solutions Eq.(3.11) into the total energy functional (3.10), the system of algebraic equations

$$
\left(\boldsymbol{K}-\omega^{2} \boldsymbol{M}\right) \tilde{\boldsymbol{u}}=\boldsymbol{F}
$$

is obtained, where $\boldsymbol{K}$ is the stiffness matrix, $\boldsymbol{M}$ is the mass matrix, $\tilde{\boldsymbol{u}}$ is the column vector of unknown displacements at the nodes, and $\boldsymbol{F}$ is the force vector. To reduce the volume of the present paper the explicit forms of the matrices and vectors in Eq.(3.12) are not given here. Note that their explicit forms are directly derived from Eq.(3.10) by employing the corresponding procedure.

\section{Numerical findings and discussions}

The domain covered by the considered body is divided into 200 parts of equal length in the direction of the $O x_{1}$ - axis and 25 parts of equal length in the direction of the $O x_{2}$ - axis. In this case, 40902 NDOFs have been obtained in total. Introduce the notations $e_{r}=e^{(l)} / e^{(r)}$, where $e^{(\bullet)}$ denotes Young's modulus of the corresponding layer.

To prove the trustworthiness of the algorithm and programs developed for the current mathematical modelling, the case where $m=2, h_{1}=h_{2}=h / 2, e_{r}=1, v^{(1)}=v^{(2)}=0.33, \eta^{(1)}=\eta^{(2)}=0, \Omega=0$ and $\alpha=\pi / 2$ is taken into account. It should be noted that the problem associated with the plate with infinite length in this case was solved by employing the Fourier integral transformation method in [12]. According to the foregoing mechanical considerations, the numerical results obtained by the use of the FEM presented in this paper must converge to the corresponding ones given by Uflyand in [12], as $h / 2 a \rightarrow 0$. This prediction is clearly demonstrated by the graphs given in Fig.2, which display the variation of the stress $\sigma_{22} h / p_{0}$ with 
respect to $x_{1} / h$ on the surface between the plate-strip and rigid foundation. The starred graph in Fig.2 indicates the one given by Uflyand [12]. So, the desired validity and trustiness of the algorithm and programs used are proved.

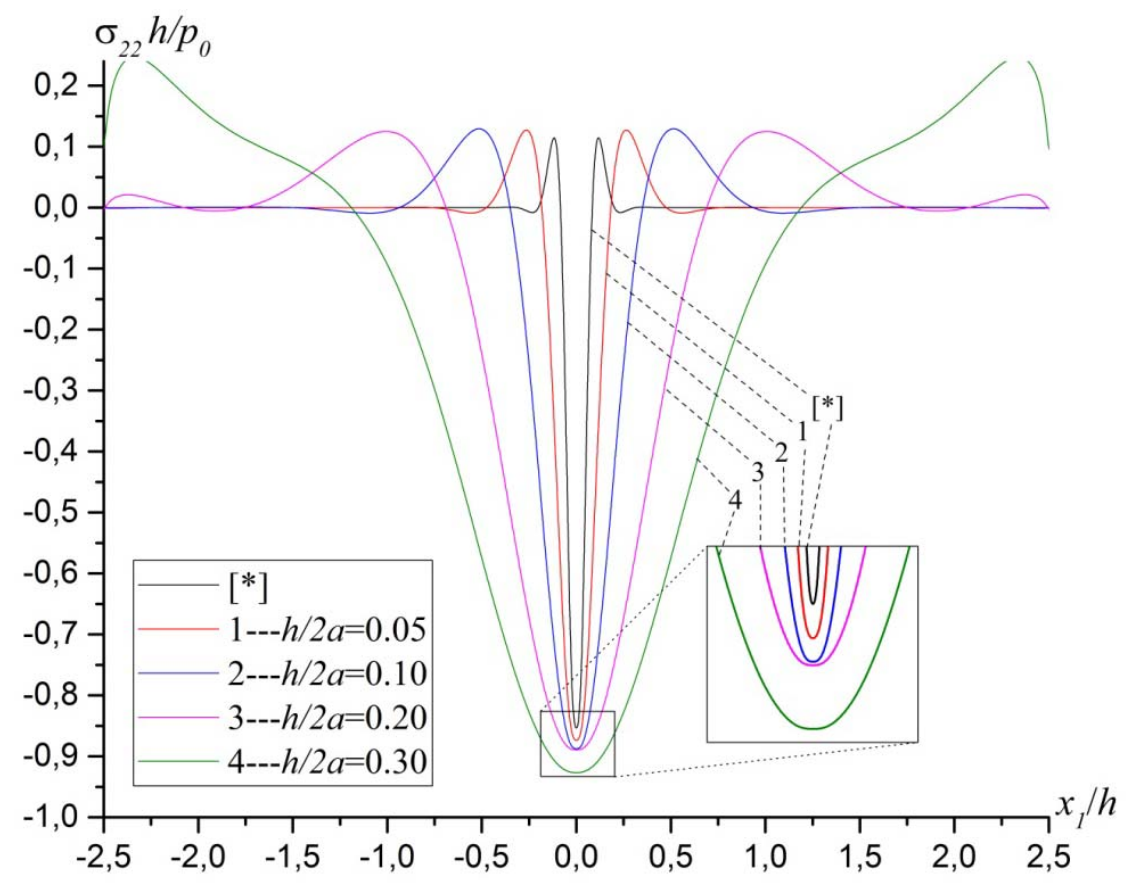

Fig.2. The distribution of $\sigma_{22} h / p_{0}$ with respect to the line $x_{1} / h$ for various thickness ratio.

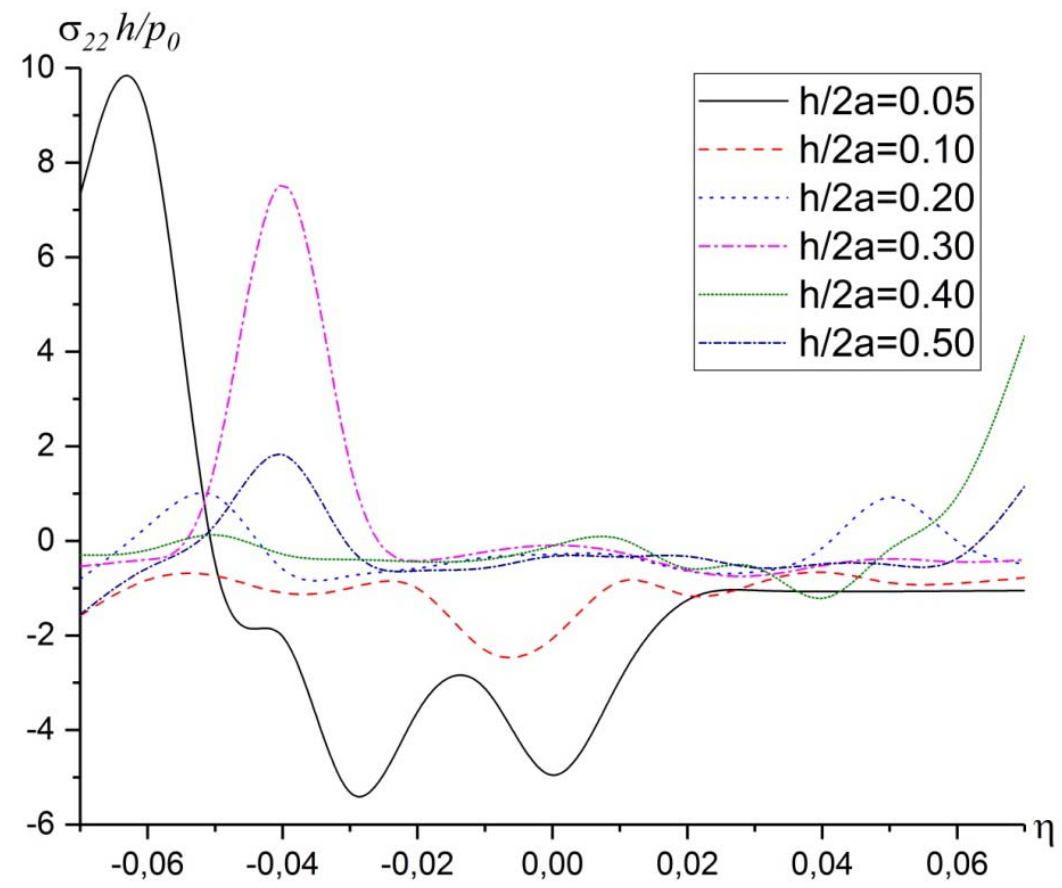

Fig.3a. The dependence between $\sigma_{22} h / p_{0}$ and $\eta$ for various thickness ratio under Case I and $\alpha=\pi / 6$. 


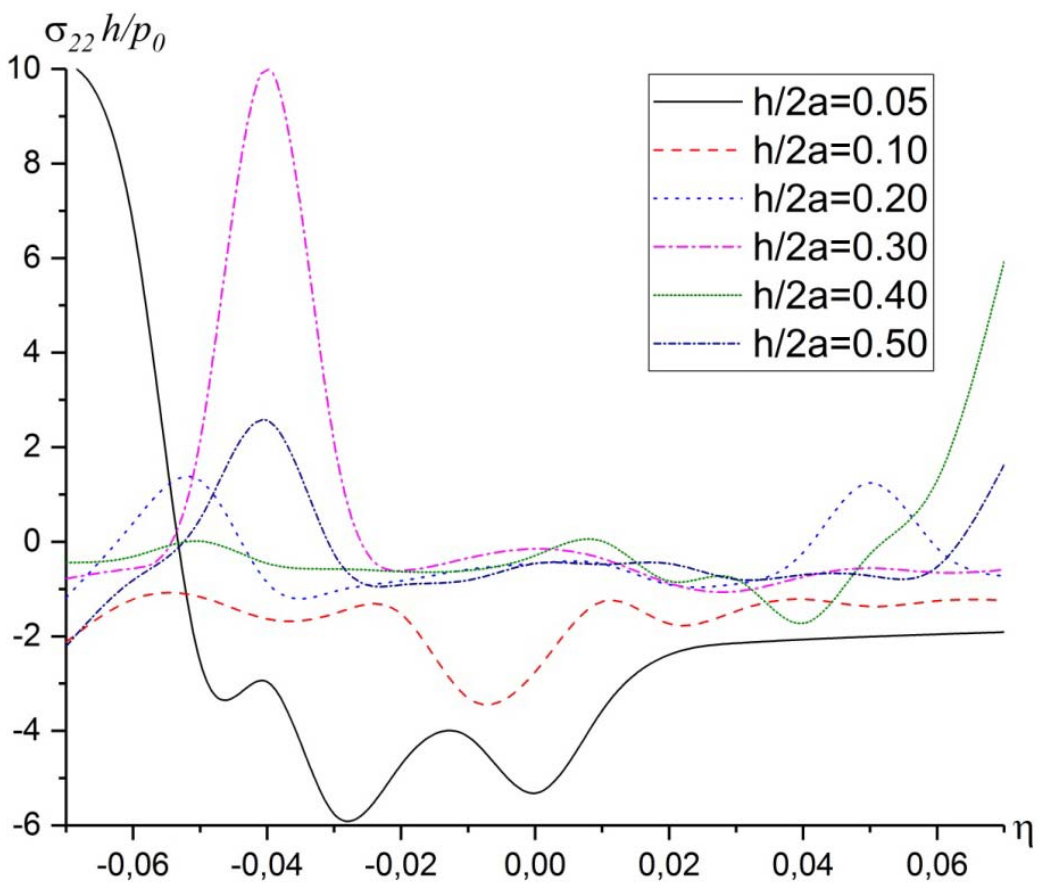

Fig.3b. The dependence between $\sigma_{22} h / p_{0}$ and $\eta$ for various thickness ratio under Case I and $\alpha=\pi / 4$.

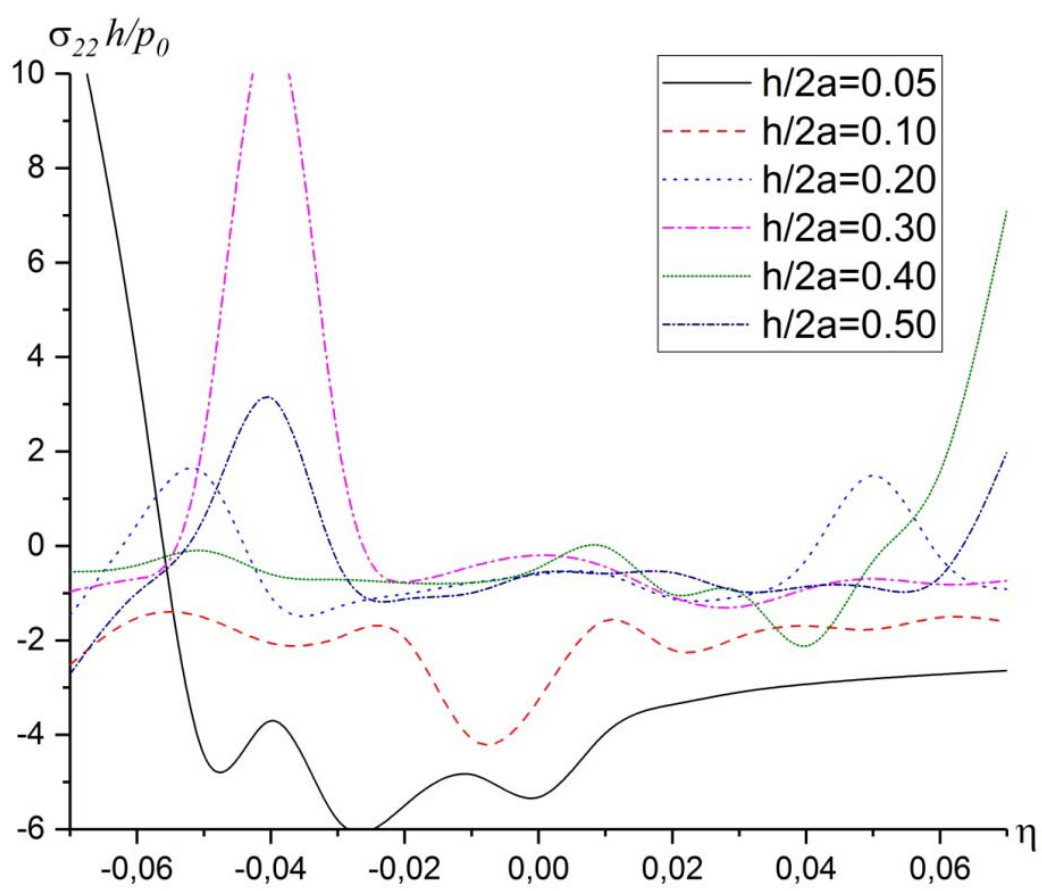

Fig.3c. The dependence between $\sigma_{22} h / p_{0}$ and $\eta$ for various thickness ratio under Case I and $\alpha=\pi / 3$. 


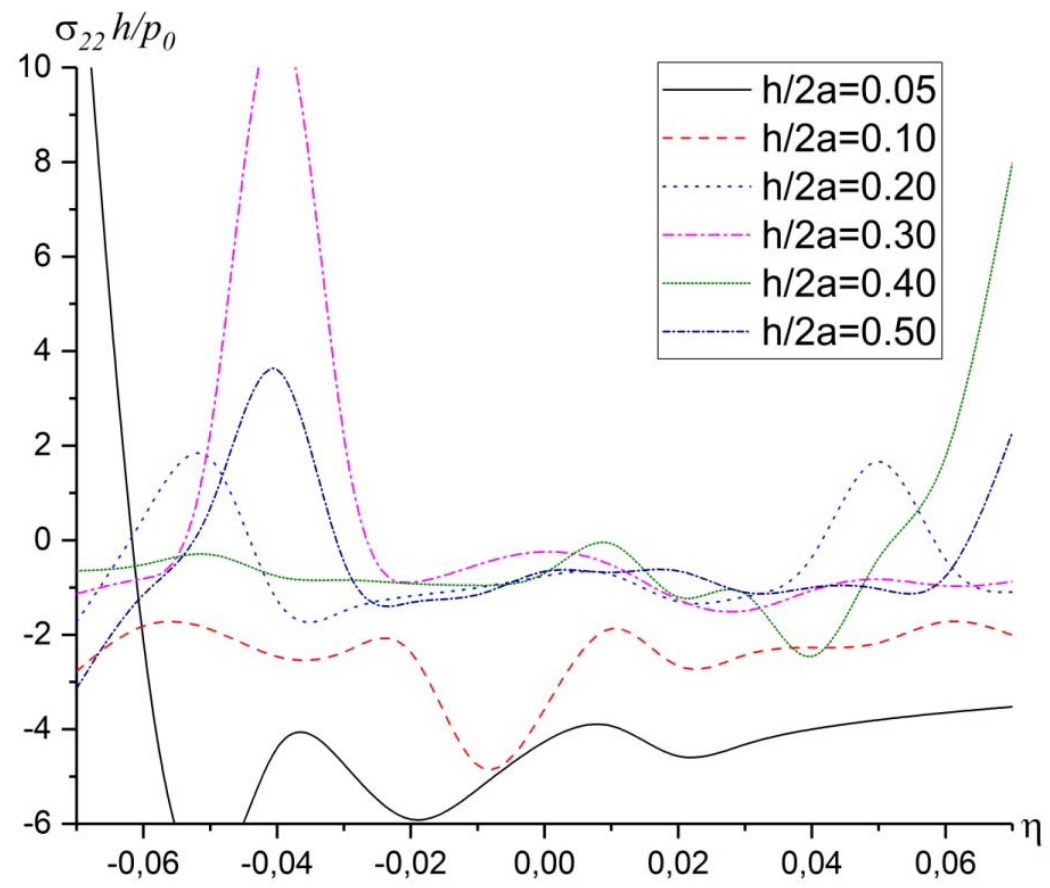

Fig.3d. The dependence between $\sigma_{22} h / p_{0}$ and $\eta$ for various thickness ratio under Case I and $\alpha=\pi / 2$.

To investigate the concrete examples, aluminum (shortly Al) with properties $v^{(A l)}=0.35$ and $\rho^{(A l)}=2.7 \times 10^{3} \mathrm{~kg} / \mathrm{m}^{3}$ and steel (shortly St) with properties $v^{(S t)}=0.29$ and $\rho^{(\mathrm{Al})}=7.86 \times 10^{3} \mathrm{~kg} / \mathrm{m}^{3}$ are selected. The considered problem addresses a very wide area. However, to present the numerical results, the following cases are only considered: Case I: $\mathrm{Al}+\mathrm{St}+\mathrm{Al}$ and Case II: $\mathrm{St}+\mathrm{Al}+\mathrm{St}$. Other desired cases can be investigated by employing the algorithm. Throughout the paper, all the investigations are made under the assumptions $h / 2 a=0.2, h_{1}=h_{2}=h_{3}, \eta^{(l)}=\eta^{(2)}=\eta^{(3)}=\eta, \Omega=0, \eta=0$ and $\alpha=\pi / 2$ at the point $(-1,0)$, unless otherwise specified.

The dependences between $\sigma_{22} h / p_{0}$ and $\eta$ are given in Fig.3 for Case I and in Fig.4 for Case II. Moreover, while these graphs are constructed, the cases where $\alpha=\pi / 6$ (Figs 3a and 4a), $\alpha=\pi / 4$ (Figs $3 b$ and $4 \mathrm{~b}$ ), $\alpha=\pi / 3$ (Figs $3 \mathrm{c}$ and $4 \mathrm{c}$ ) and $\alpha=\pi / 2$ (Figs 3d and 4d) are taken into account. The absolute values of the normal stress $\sigma_{22} h / p_{0}$ decrease (increase) with the initial stretching (compressing) parameter and with the angle $\alpha$. But, its absolute values increase with decreasing $h / 2 a$. The distributions of the graphs given for Case II are more stable than the ones for Case I. Moreover, for Case I, the initial compressing applied to the layers causes the oscillating character of the distribution of $\sigma_{22} h / p_{0}$ to become more sensitive. However, the initial stretching increases the stability of the system. As can be seen from a comparison of the graphs in Figs 3 and 4, the stress $\sigma_{22} h / p_{0}$ depends linearly on the initial stress parameter for Case II, but it does not depend linearly for Case I. This result is explained by the selection of the plates. The numerical results indicate that there exist certain locations where the parametric resonance of the normal stress $\sigma_{22} h / p_{0}$ occurs for certain values of the initial stress parameter $\eta$. The case where $h / 2 a=0.3$ and $\eta=0.01$ in Fig. $4 \mathrm{~b}$ can be given as an example. The numbers of the local maximums and minimums of $\sigma_{22} h / p_{0}$ decrease with $h / 2 a$ and with $\alpha$. An increase in the values of $h / 2 a$ causes to decrease (increase) the influence of the initial stretching (compressing) on the distribution of $\sigma_{22} h / p_{0}$. 


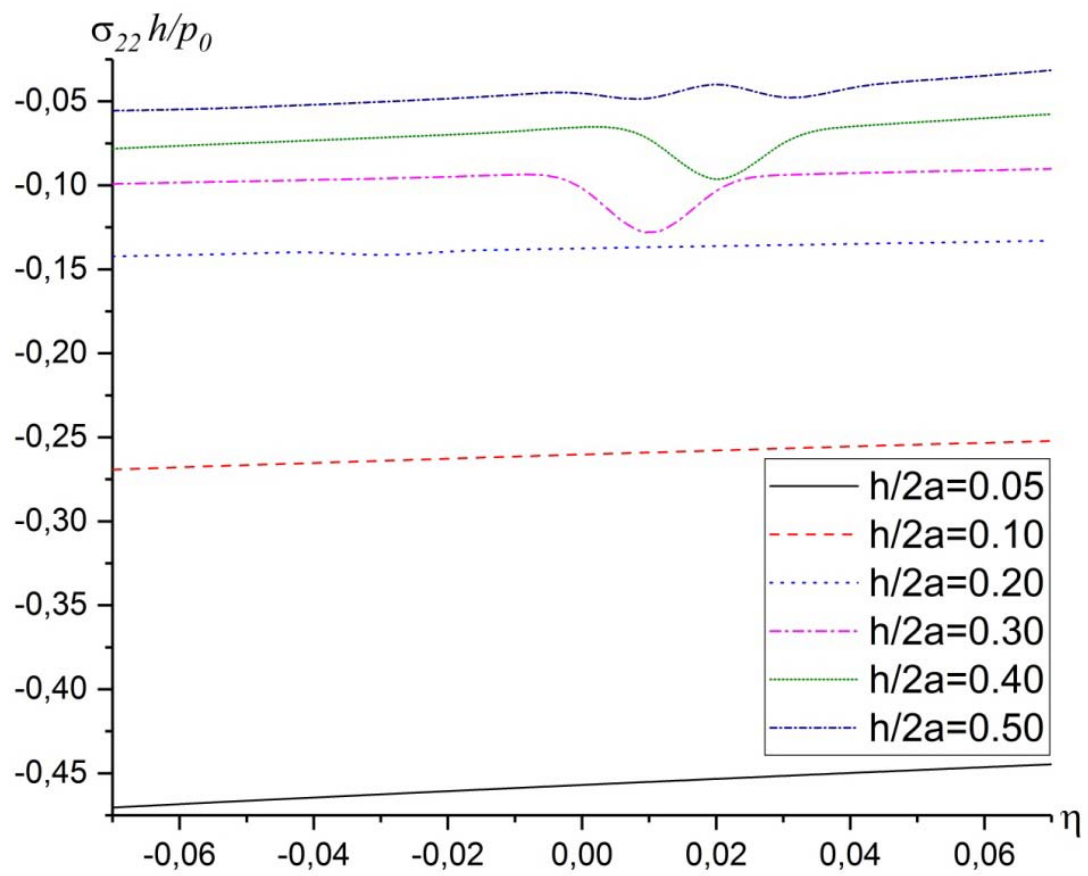

Fig.4a. The dependence between $\sigma_{22} h / p_{0}$ and $\eta$ for various thickness ratio under Case II and $\alpha=\pi / 6$.

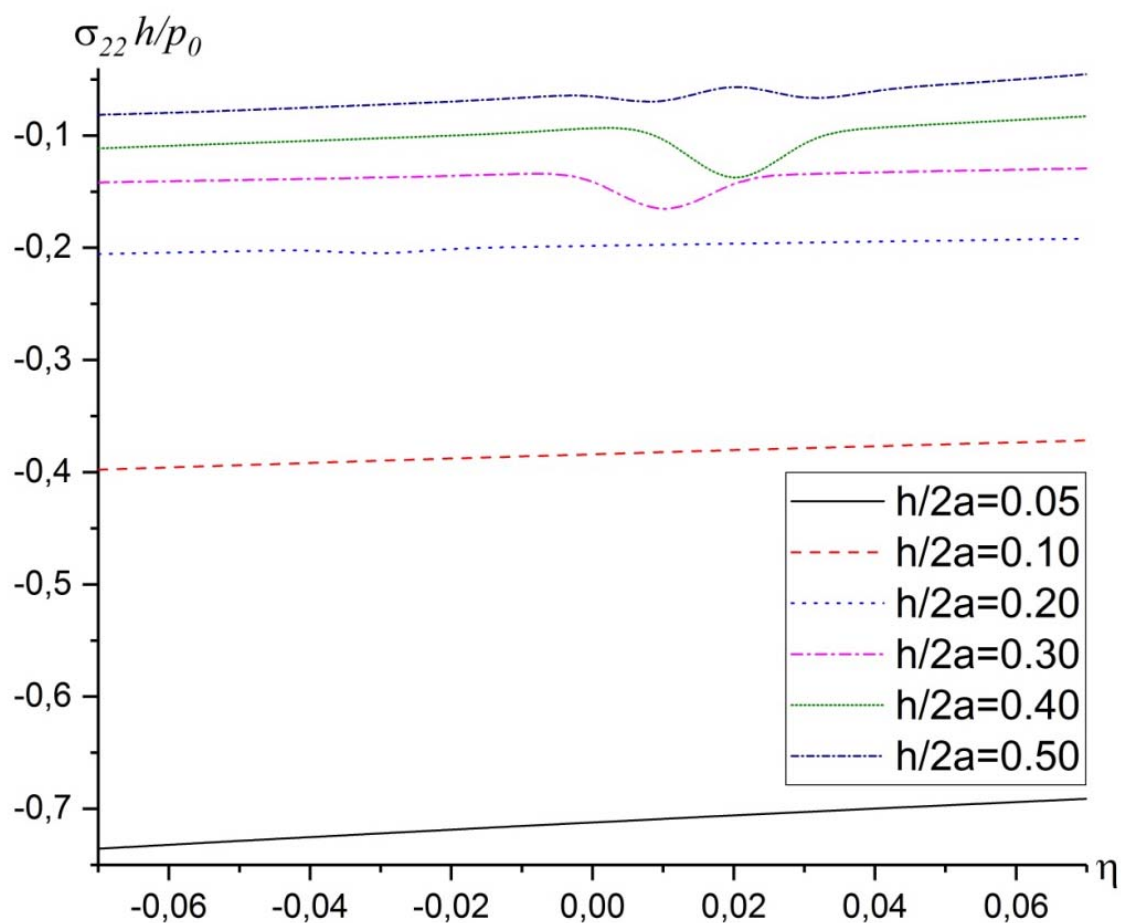

Fig.4b. The dependence between $\sigma_{22} h / p_{0}$ and $\eta$ for various thickness ratio under Case II and $\alpha=\pi / 4$. 


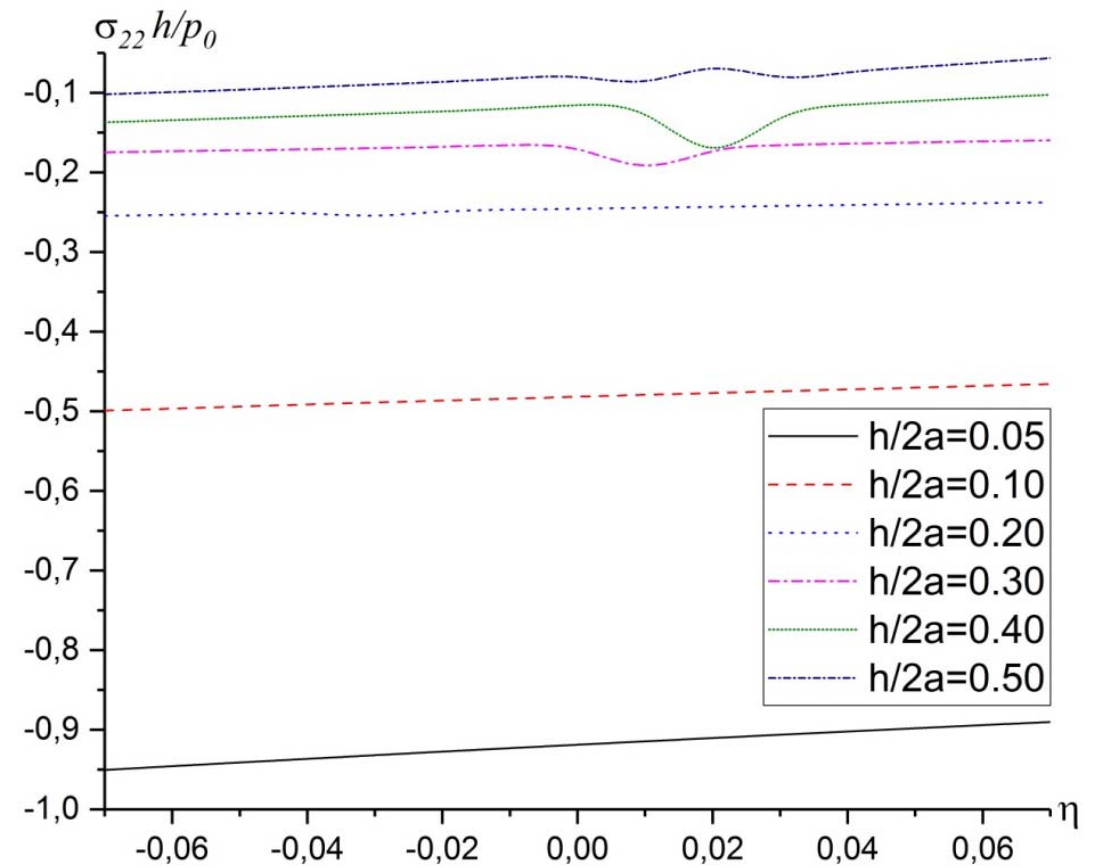

Fig.4c. The dependence between $\sigma_{22} h / p_{0}$ and $\eta$ for various thickness ratio under Case II and $\alpha=\pi / 3$.

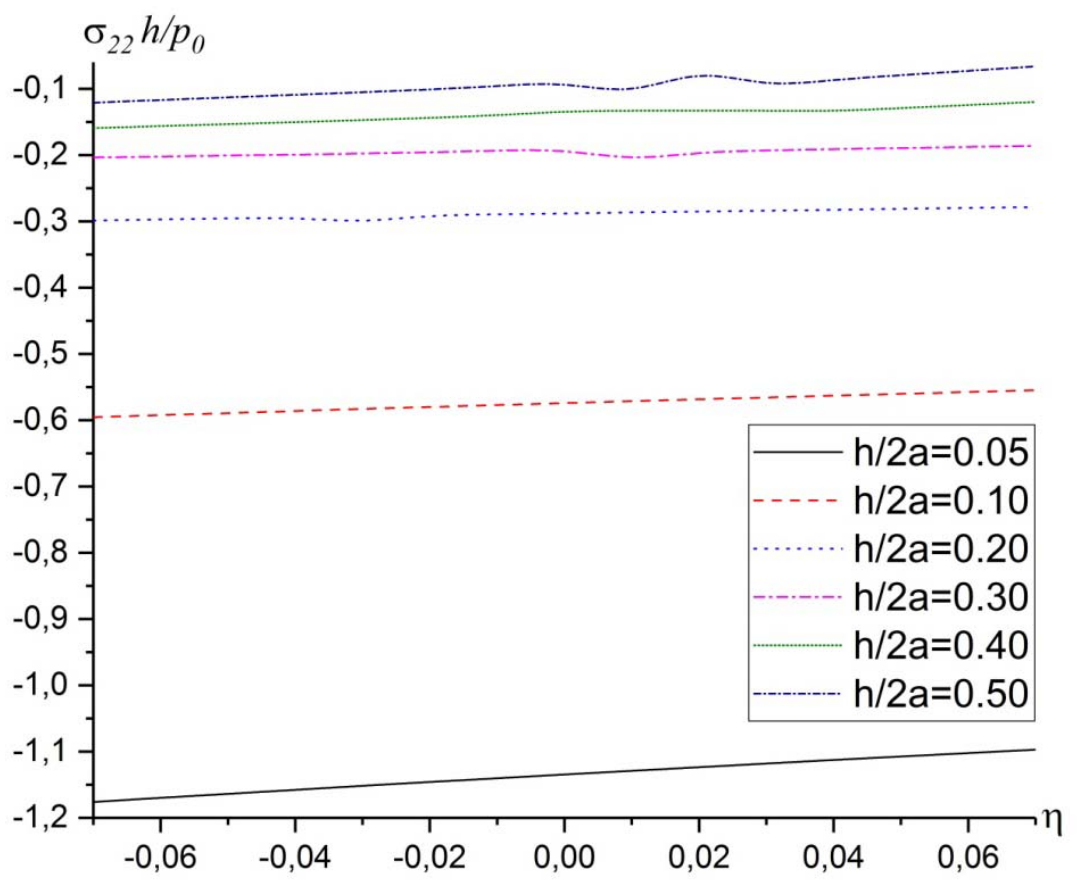

Fig.4d. The dependence between $\sigma_{22} h / p_{0}$ and $\eta$ for various thickness ratio under Case II and $\alpha=\pi / 2$.

Figures 5 and 6 display the influence of the initial stress parameter $\eta$ on the frequency response of the normal stress $\sigma_{22} h / p_{0}$ for Case I and Case II, respectively. Note that these figures are plotted for the cases where $\alpha=\pi / 6$ (Figs 5a and 6a), $\alpha=\pi / 4$ (Figs 5b and 6b), and $\alpha=\pi / 3$ (Figs 5c and 6c) and $\alpha=\pi / 2$ (Figs $5 \mathrm{~d}$ and $6 \mathrm{~d}$ ). The absolute values of $\sigma_{22} h / p_{0}$ decrease with $\Omega$. It follows from the graphs that there exist locations where $\sigma_{22} h / p_{0}$ reaches the extrema for certain values of $\Omega$. These values are 
called the resonance values and denoted by $\Omega^{*}$. As can be seen from the graphs, the resonance values of $\sigma_{22} h / p_{0}$ decrease with (increasing) the initial stretching (compressing) parameter. The numbers of the local maximums and minimums of the $\sigma_{22} h / p_{0}$ increase with (increasing) the initial stretching (compressing) parameter. It is concluded from the graphs that the influence of the initial stress parameter $\eta$ on the frequency response of the stress $\sigma_{22} h / p_{0}$ is considerable not only in the quantitative sense, but also in the qualitative sense. As in Figs 3 and 4, the system becomes quite stable for the second case. The numerical results indicate that the layer adhered to the rigid foundation should be selected as stiffer than the others.

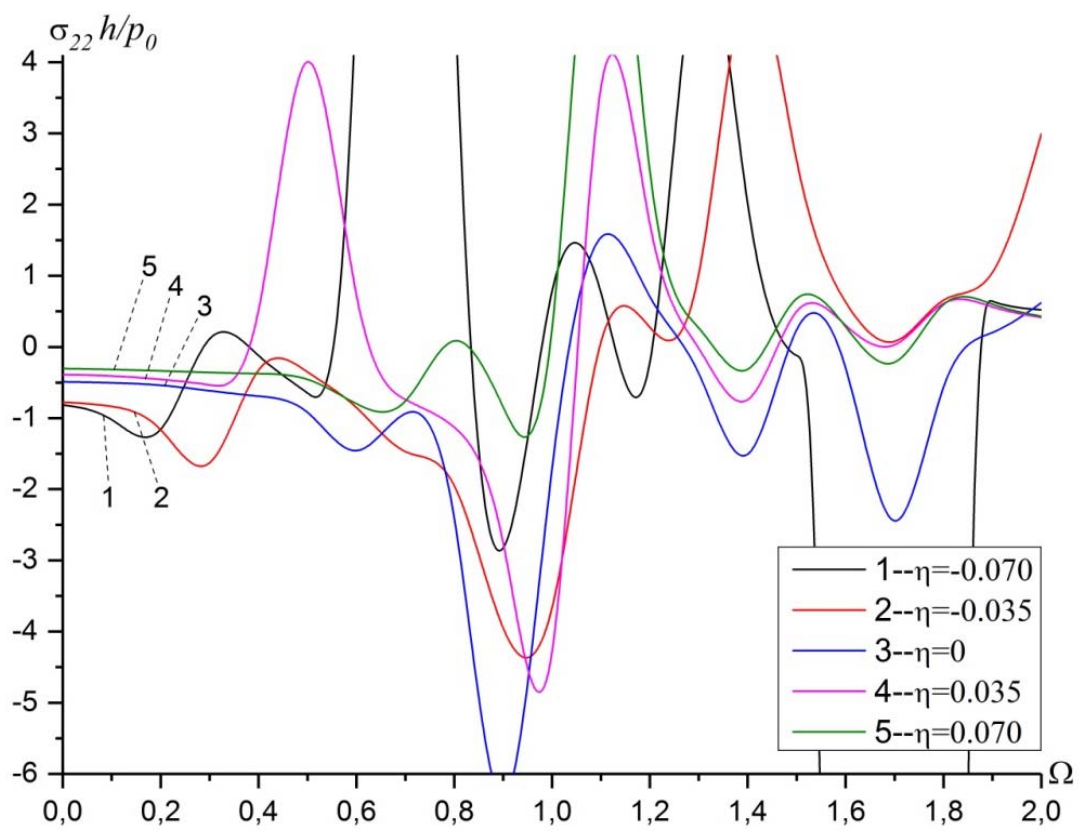

Fig.5a. The dependence between $\sigma_{22} h / p_{0}$ and $\Omega$ for various values of $\eta$ under Case I and $\alpha=\pi / 6$.

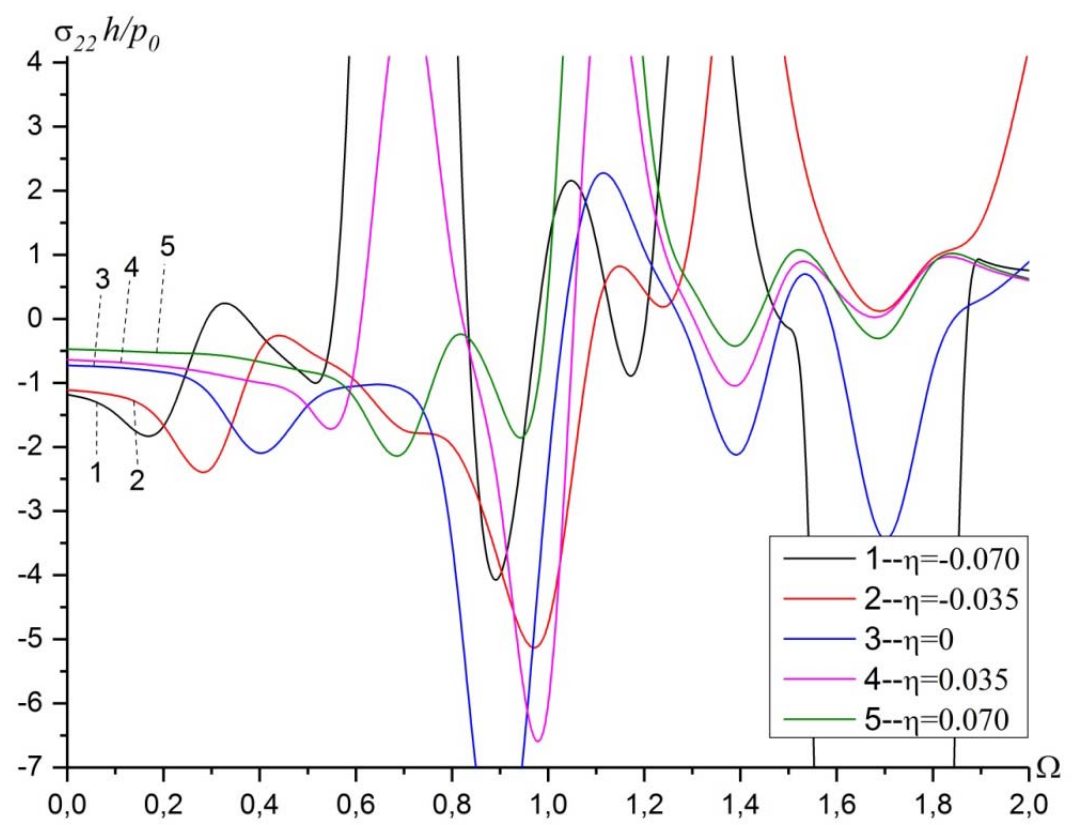

Fig.5b. The dependence between $\sigma_{22} h / p_{0}$ and $\Omega$ for various values of $\eta$ under Case I and $\alpha=\pi / 4$. 


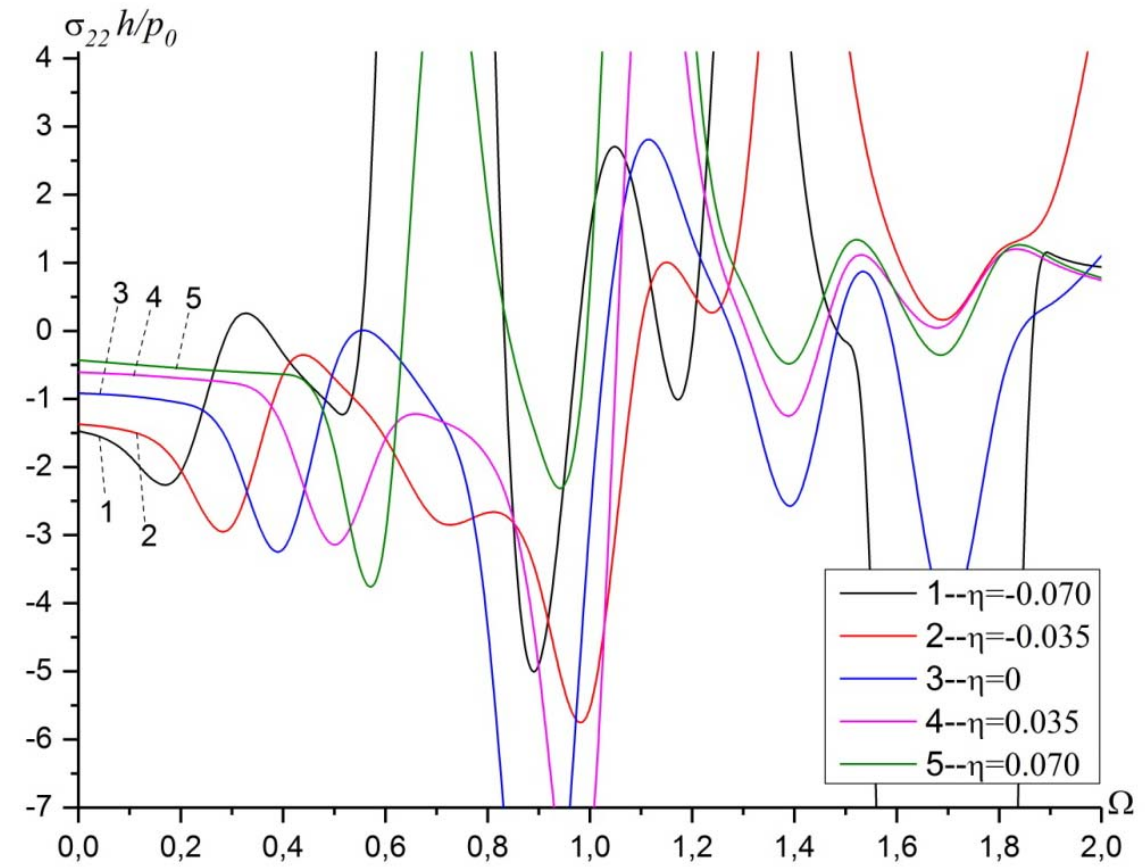

Fig.5c. The dependence between $\sigma_{22} h / p_{0}$ and $\Omega$ for various values of $\eta$ under Case I and $\alpha=\pi / 3$.

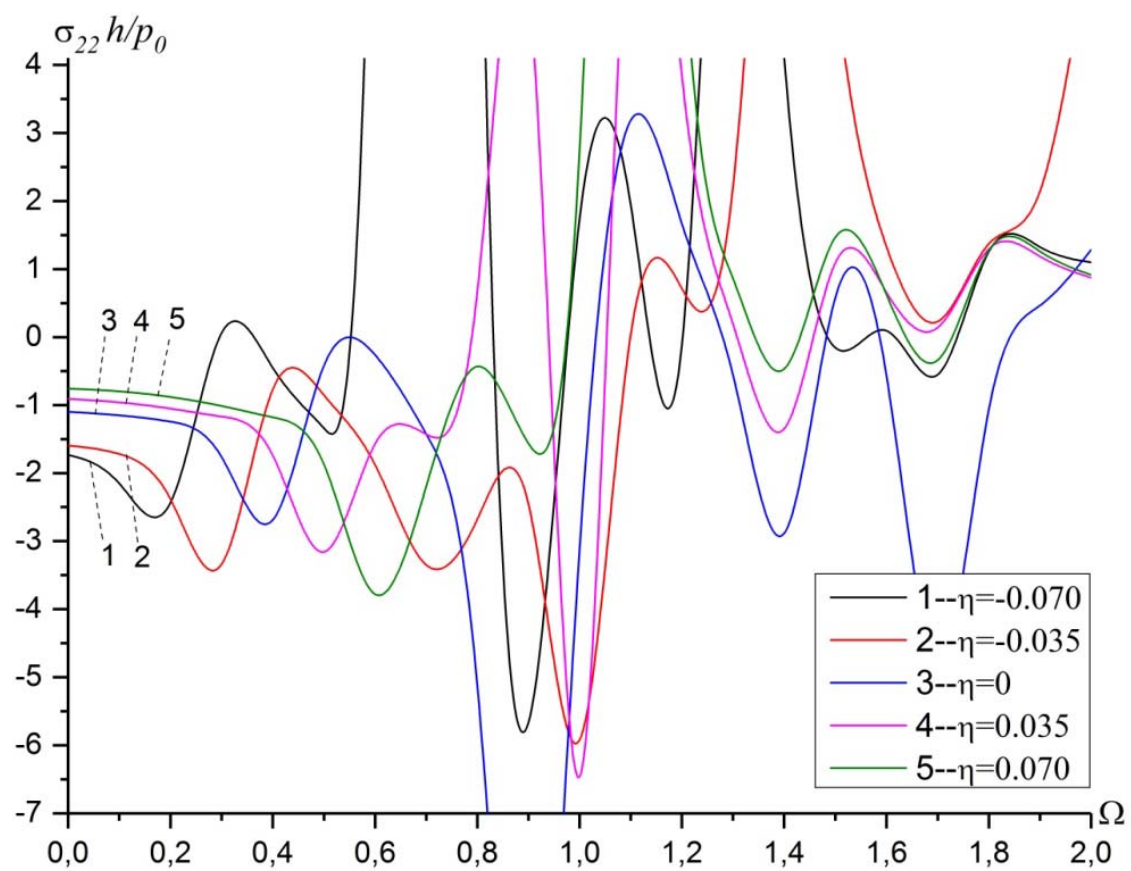

Fig.5d. The dependence between $\sigma_{22} h / p_{0}$ and $\Omega$ for various values of $\eta$ under Case I and $\alpha=\pi / 2$. 


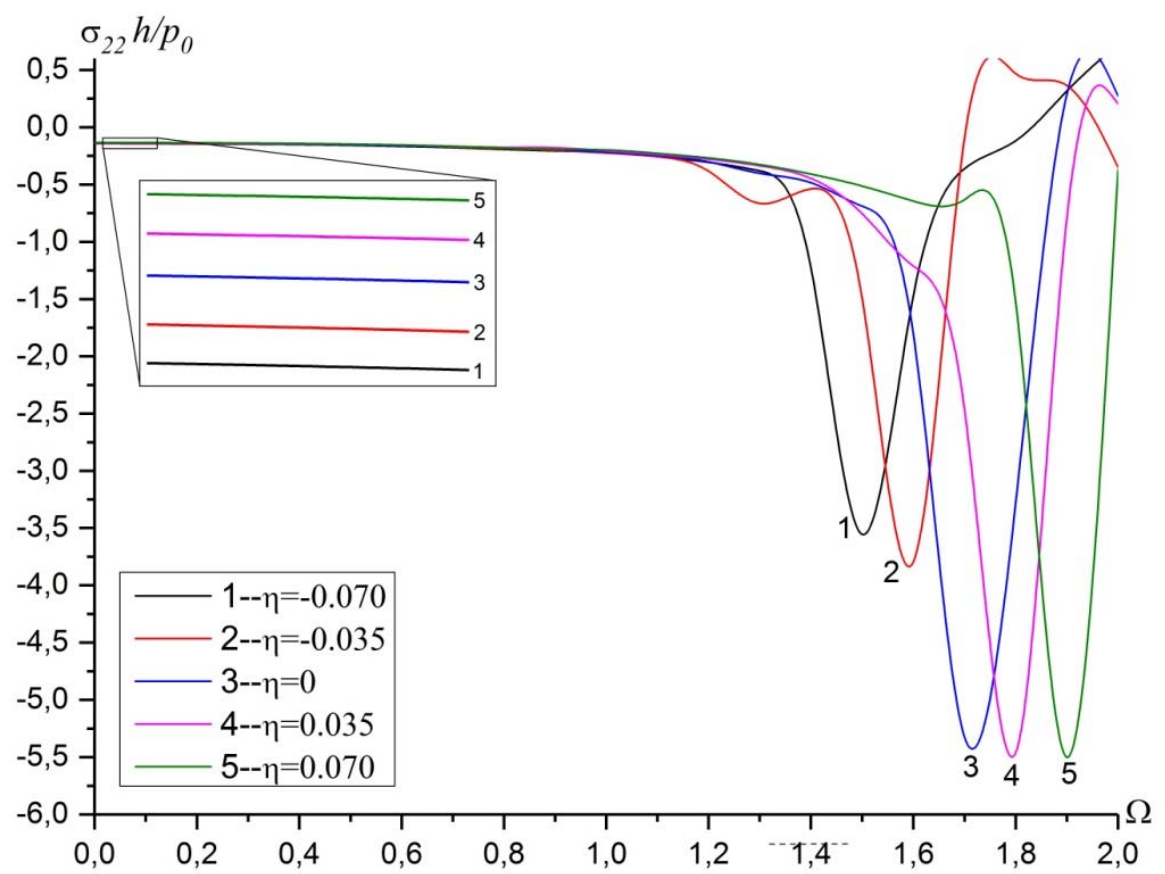

Fig.6a. The dependence between $\sigma_{22} h / p_{0}$ and $\Omega$ for various values of $\eta$ under Case II and $\alpha=\pi / 6$.

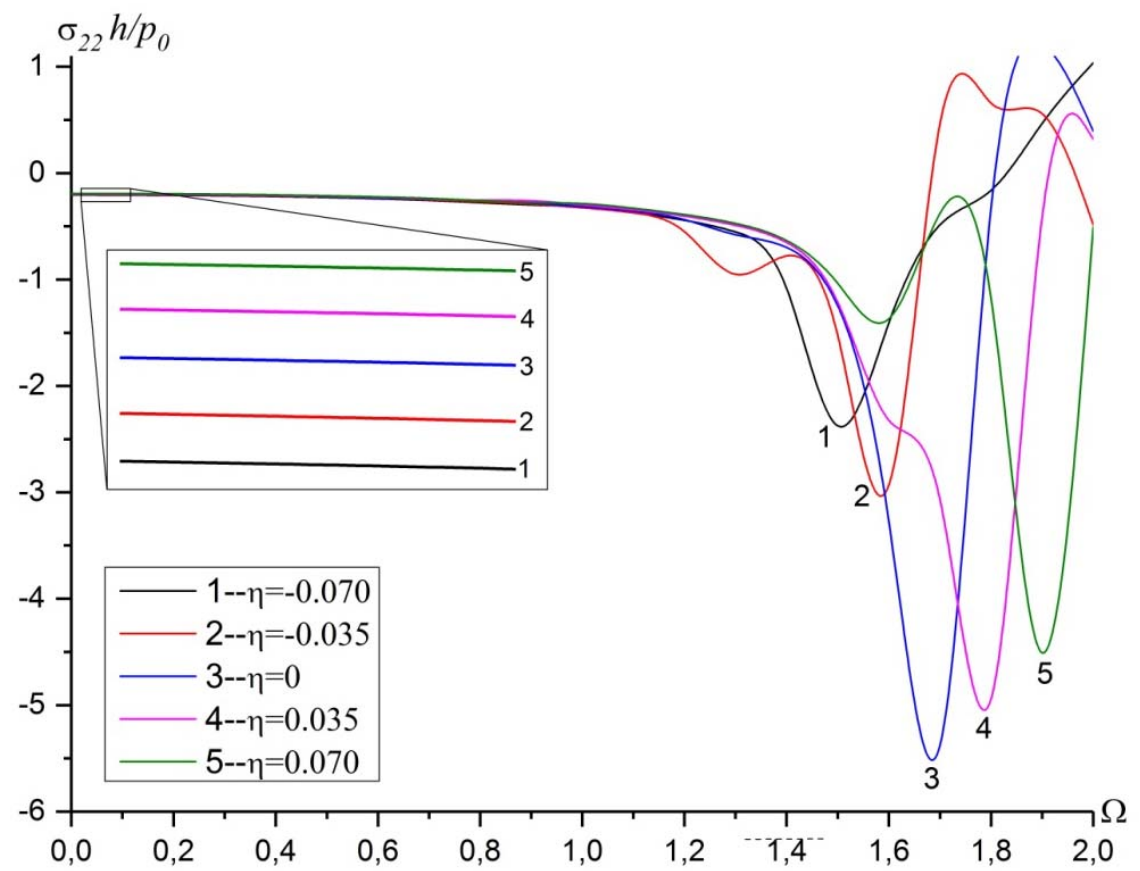

Fig.6b. The dependence between $\sigma_{22} h / p_{0}$ and $\Omega$ for various values of $\eta$ under Case II and $\alpha=\pi / 4$. 


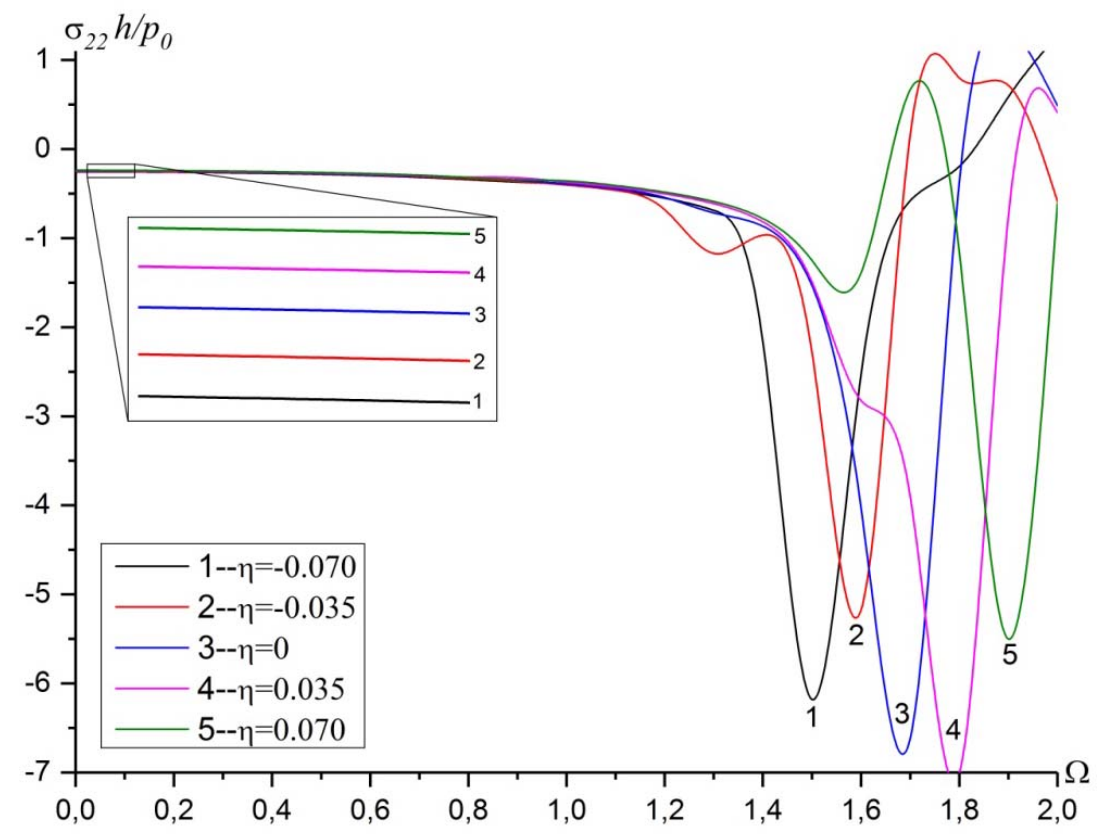

Fig.6c. The dependence between $\sigma_{22} h / p_{0}$ and $\Omega$ for various values of $\eta$ under Case II and $\alpha=\pi / 3$.

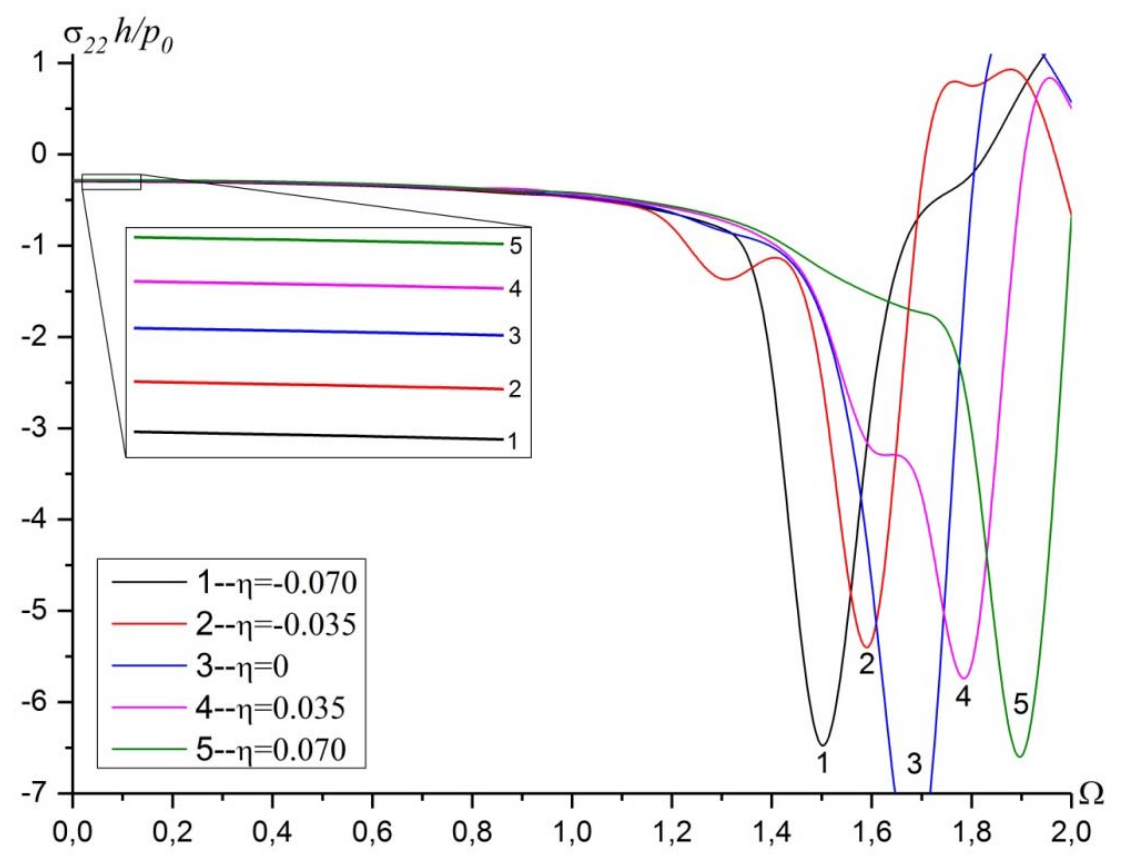

Fig.6d. The dependence between $\sigma_{22} h / p_{0}$ and $\Omega$ for various values of $\eta$ under Case II and $\alpha=\pi / 2$.

\section{Nomenclature}

$a$ - the length of the plate-strip

$a_{i}^{(k)}\left(b_{i}^{(k)}\right)$ - a nodal displacement of the $i$ th node of the $k$ th element in the direction of $O x_{1}\left(O x_{2}\right)$ 


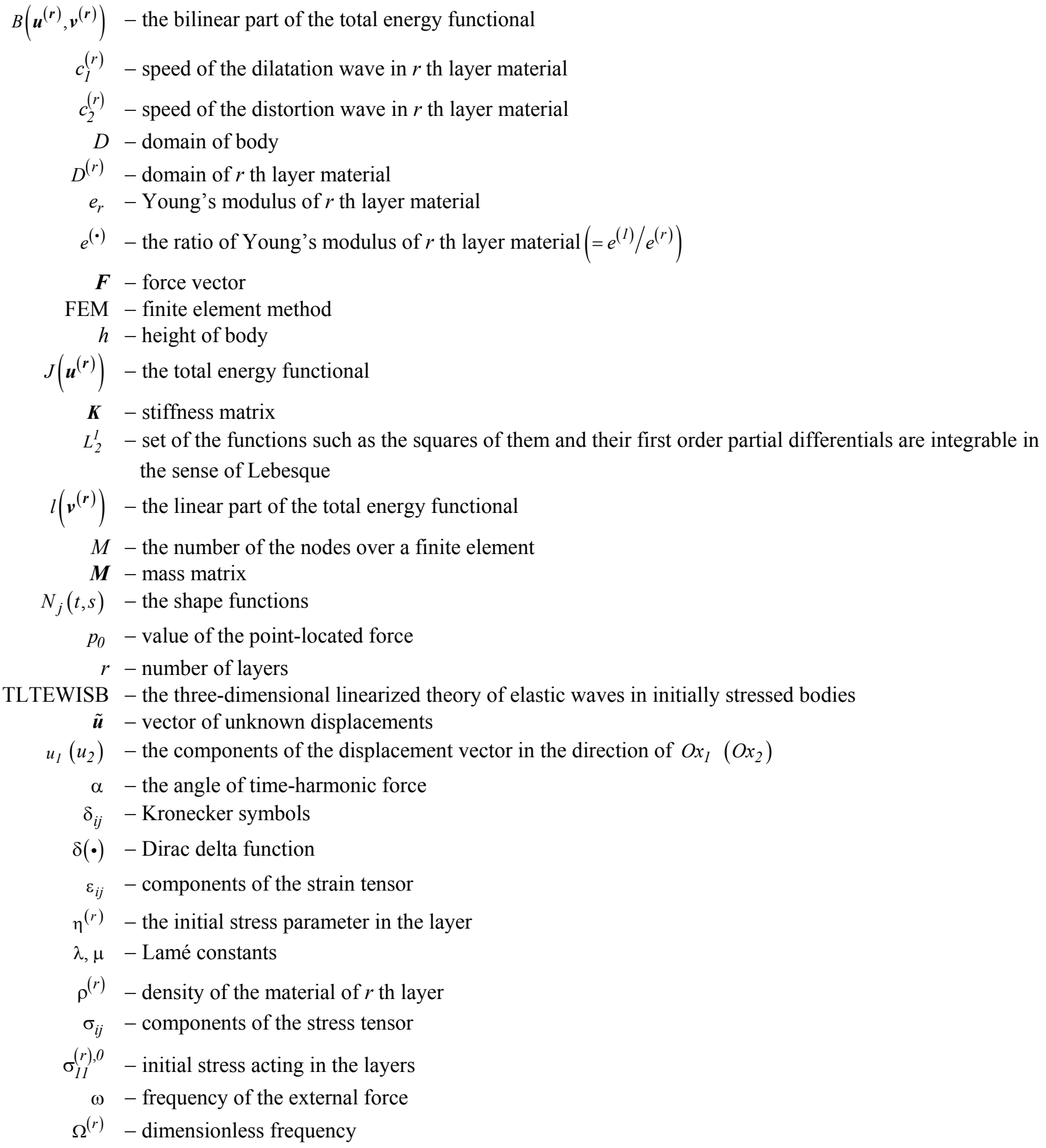

\section{References}

[1] Guz A.N. (1986): Elastic Waves in a Body Initial Stresses, I. General Theory. - Kiev: Naukova Dumka (in Russian).

[2] Guz A.N. (1986): Elastic Waves in a Body Initial Stresses, II. Propagation Laws. - Kiev: Naukova Dumka (in Russian).

[3] Zamanov A.D. (2001): Stress distribution in a rigidly clamped composite plate with locally curved structures under forced vibration. - Internat. Appl. Mech., vol.37, No.9, pp.1189-1195. 
[4] Akbarov S.D. and Ozaydin O. (2001): The effect of initial stresses on harmonic fields with the stratified half plane. - Eur. J. Mech. A/Solids, vol.20, pp.385-396.

[5] Nayak A.K., Moy S.S.J. and Shenoi R.A. (2002): Free vibration analysis of composite sandwich plates based on Reddy's higher-order theory. - Composites: Part B, vol.33, No.7, pp.505-519.

[6] Zhuk Y.A. and Guz I.A. (2006): Influence of prestress on the velocities of plane waves propagating normally to the layers of nanocomposites. - Int. Appl. Mech., vol.42, No.7, pp.729-743.

[7] Zhuk Y.A. and Guz I.A. (2007): Features of propagation of plane waves along to the layers of an initially stressed nanocomposite material. - Int. Appl. Mech., vol.43, No.4, pp.3-26

[8] Pandit M.K., Singh B.N. and Sheikh A.H. (2008): Buckling of laminated sandwich plates with soft core based on an improved higher order zigzag theory. - Thin Wall Struct., vol.46, No.11, pp.1183-1191.

[9] Cilli A. and Ozturk A. (2010): Dispersion of torsional waves in initially stressed multilayered circular cylinders. Mech. Compos. Mater., vol.46, No.2, pp.227-236.

[10] Wen-tao H., Tang-dai X. and Wei-yun C. (2014): Influence of lateral initial pressure on axisymmetric wave propagation in hollow cylinder based on first power hypo-elastic model. - J. Cent. South Univ., vol.21, pp.753760.

[11] Hutton D. (2004): Fundamentals of Finite Element Analysis. - New York: McGraw-Hills.

[12] Uflyand Y.S. (1963): Integral Transformations in the Theory of Elasticity. - Moscow-Leningrad: Nauka.

Received: January 27, 2017

Revised: June 9, 2017 